\title{
Is placental KISS-1 expression associated with first trimester abortion spontaneous?
}

\author{
Eser Colak, MD¹, Emel Ebru Ozcimen, MD¹, Ozgur Hilal Erinanç, MD², Yusuf Aytac Tohma, MD³, \\ Mehmet Ufuk Ceran, MD ${ }^{1}$ \\ Departments of ${ }^{1}$ Gynecology and Obstetrics, ${ }^{2}$ Pathology, Konya Medical and Research Center, Baskent University School of Medicine, Konya; \\ ${ }^{3}$ Department of Gynecology and Obstetrics, Baskent University School of Medicine, Ankara, Turkey
}

\section{Objective}

Several studies have examined biological markers during the first trimester to predict the maintenance of a healthy pregnancy. One such marker is kisspeptin, which is encoded by the KISS-1 gene. We aimed to determine whether firsttrimester pregnancy losses were associated with levels of placental KISS-1 expression.

\section{Methods}

This prospective case control study was conducted at a tertiary center. The study group included 27 and 24 patients who underwent dilation and curettage at $<10$ weeks of gestation, due to first trimester spontaneous pregnancy loss and for elective termination (control), respectively. Placental and decidual tissues from all patients were sectioned and immunohistochemically analyzed for kisspeptin.

\section{Results}

Age, gravida status, parity number, gestational week, and number of previous abortions did not significantly differ between the groups. KISS-1 expression levels were significantly lower in the group with spontaneous abortion compared with the group with elective termination. The median staining intensity of KISS-1 expression in the elective and spontaneous termination groups were 3 (strong) and 2 (moderate), respectively $(P=0.004)$. KISS- 1 expression levels were significantly lower among patients with previous abortions in the elective termination group $(P=0.002)$.

\section{Conclusion}

KISS-1 expression levels were found to be significantly reduced in patients with spontaneous pregnancy loss; KISS-1 plays an important role in the implantation and continuation of pregnancy.

Keywords: Genes, tumor suppressor; Abortion, spontaneous; Pregnancy, unwanted; Placenta

\section{Introduction}

The first trimester of pregnancy is a critical period for the continuity and progression of a healthy pregnancy. Approximately $15 \%$ of clinically confirmed pregnancies result in spontaneous abortion, and $80 \%$ of those occur during the first trimester of pregnancy [1]. The major etiological factors for spontaneous abortions during the first trimester of pregnancy are genetic, endocrine, anatomical, and immunological; however, the etiology of $50 \%$ of such abortions remains unknown. Regulation of the finely controlled process of trophoblast invasion plays a major role in preventing complications of pregnancy, including early spontaneous abortion $[2,3]$. The ability of markers such as mean arterial pressure,
Received: 2019.12.17. Revised: 2020.01.21. Accepted: 2020.02.04. Corresponding author: Eser Colak, MD

Department of Gynecology and Obstetrics, Konya Medical and Research Center, Baskent University School of Medicine, Hocacihan mah. Saray cad. No:1, Selcuklu, Konya 42030, Turkey E-mail: dresercolak@hotmail.com https://orcid.org/0000-0002-8184-7531

Articles published in Obstet Gynecol Sci are open-access, distributed under the terms of the Creative Commons Attribution Non-Commercial License (http://creativecommons org/licenses/by-nc/3.0/) which permits unrestricted non-commercial use, distribution, and reproduction in any medium, provided the original work is properly cited.

Copyright $\odot 2020$ Korean Society of Obstetrics and Gynecology 


\section{Obstetrics \& Gynecology Science}

Eser Colak, et al. Placental KISS-1 in first trimester

uterine artery pulsatility index, placental growth factor, pregnancy-associated plasma protein $A$, maternal serum alpha-fetoprotein, and free beta-HCG, in predicting healthy maintenance of pregnancy, have been examined during the first trimester $[4,5]$. However, studies of markers that could indicate the well-being of pregnancy during the first trimester, are under way.

Recent studies have focused on molecular mechanisms like those of tumor cells because trophoblast migration and invasion occur via such molecular mechanisms $[6,7]$. One such mechanism is that of kisspeptin.

Kisspeptin constitutes a group of peptide fragments encoded by the KISS-1 gene (a tumor metastasis suppressor) in humans and each fragment acts on various tissues via numerous mechanisms [8]. Kisspeptin-10 (KP-10) might play a role in the regulation of trophoblast invasion, puberty onset, and in the development of the hypothalamic-pituitary-gonadal axis $[9,10]$. Kisspeptins, especially KP-10, reportedly play important roles in trophoblast invasion through the regulation of placental angiogenesis. Trophoblast invasiveness reduced by KP-10 might induce the downregulation of matrix metallopeptidase activity [11]. Kisspeptin expression in syncytiotrophoblasts and cytotrophoblasts is reduced in women who experience recurrent spontaneous abortion compared with controls [12]. Sullivan-Pyke et al. [13] found lower serum kisspeptin levels in cases experiencing spontaneous abortions. Thus, kisspeptin might serve as a new serum biomarker to discriminate the likelihood of spontaneous abortion from viable intrauterine pregnancy [13].

The present study compared the expression of placental KISS-1 that encodes kisspeptin, between first-trimester pregnancy losses and elective pregnancy terminations to determine the differences at the tissue level.

\section{Materials and methods}

\section{Study population}

This prospective, case control study was conducted at the Department of Gynecology and Obstetrics of a tertiary center.

The sample size of the study was calculated using the G*Power (G*Power Ver. 3.1.9.2, Franz Faul, Üniversität zu Kiel, Kiel, Germany) statistical package. The required sample sizes for $a=0.05$ and $Z a=1.96$, and $\beta=0.10$ and $Z \beta=-1.28$ was calculated for each group. The study and control groups included 27 and 24 consecutive patients who underwent dilation and curettage at $<10$ weeks of gestation due to first trimester spontaneous pregnancy loss and for elective termination, respectively, between July 2017 and January 2018 . Elective pregnancy termination is possible before gestational week 10 in Turkey. Therefore, we included first-trimester pregnancy losses only before gestational week 10 .

Spontaneous pregnancy loss was diagnosed via transvaginal ultrasound and clinical assessment. We excluded patients with a retroplacental hematoma, active maternal infection, systemic lupus erythematosus, hypertension, diabetes, chronic renal insufficiency, alcohol consumption, a smoking habit (cigarettes), or a history of $\geq 2$ spontaneous abortions. According to the guidelines of the Royal College of Obstetricians and Gynaecologists, recurrent pregnancy loss is defined as a total of $\geq 3$ abortions [14]. We analyzed the karyotypes of all patients, and excluded those with abnormalities to unmask and avoid possible effects of chromosomal abnormalities on kisspeptin expression between the groups. Patients with vaginal bleeding and hematomas were not included due to low risk. As much as possible, we aimed to select only patients with healthy pregnancies for the control group.

Curettage specimens from 27 spontaneous pregnancy losses and 24 elective pregnancy terminations histopathologically diagnosed as "decidua showing the Arias-Stella reaction and chorionic villi" at the Faculty of Medicine, Department of Pathology between 2001 and 2003 were collected and archival hematoxylin and eosin stained sections were reevaluated. Kisspeptin was immunohistochemically assessed in tissue samples containing decidua and chorionic villi without areas of hemorrhage and necrosis.

\section{Immunohistochemistry}

Sections were incubated at $56^{\circ} \mathrm{C}$ for 24 hours, deparaffinized in xylene, then rehydrated using a descending alcohol series (100-70\%). Endogenous peroxidase activity was blocked by immersing the sections for 5 minutes in methanol containing $0.5 \%$ hydrogen peroxide. Antigens were retrieved by microwave-heating the sections in trisodium citrate buffer (10 mM sodium citrate, pH 6.0) for 10 minutes. The sections were rinsed 3 times in de-ionized distilled water, immersed in $0.3 \%$ hydrogen peroxidase for 30 minutes to block endogenous peroxidase activity, then washed in PBS for 2-3 minutes. Non-specific background staining was minimized 


\title{
Obstetrics \& Gynecology Science
}

\author{
Vol. 63, No. 4, 2020
}

using Ultra V Block (Thermo Fisher Scientific Inc., Waltham, MA, USA) for 5 minutes. The sections were then incubated for 1 hour at room temperature with the mouse monoclonal primary antibody to KISS-1, 24-Q (Santa Cruz Biotechnology Inc., Dallas, TX, USA) diluted 1:100. This antibody was raised against a partial recombinant protein mapped within amino acids 46-145 of KISS-1 of human origin. After a wash with PBS for 5 minutes, Goat Anti-Polyvalent (Lab Vision Corp., Fremont, (A, USA) was applied, then the sections were washed in PBS. The sections were incubated for 15 minutes at room temperature with streptavidin peroxidase (Lab Vision Corp.). Immunoreactivity was visualized using the chromogen, DAB (Thermo Fisher Scientific Inc.). The sections were counterstained with Mayer's hematoxylin solution and mounted. Staining specificity was confirmed by comparison with a positive control.

\section{Analysis of immunohistochemical expression}

Two pathologists who were blinded to the pregnancy stage and outcomes analyzed all sections. Immunohistochemical staining was evaluated by scanning whole sections for antibodies using a BX51 light microscope (Olympus Optical Co., Ltd., Tokyo, Japan) at 4x, 10x, 20x, and 40x magnification. KISS-1 proteins were mainly located in the glandular epithelium, decidualized stromal cells, cytotrophoblasts, and syncytiotrophoblasts.

The results are expressed as ratios (\%) of positivity based on staining intensity scored as 0 (negative), 1+ (low), 2+ (moderate), and $3+$ (strong). Ratios (\%) of stained cells were: $0(<10 \%), 1(10-25 \%), 2(10-50 \%), 3(51-80 \%)$, and 4
(>80\%). The final score was calculated as the product of the ratio of stained cells and staining intensity, resulting in weak (0-2), moderate (3-6), and strong (8) KiSS-1 expression [15].

\section{Statistical analysis}

Variables were analyzed using SPSS 22.0 (IBM Corp., Armonk, NY, USA) and MedCalc 14 (MedCalc Software Ltd., Ostend, Belgium) software. The normality of data distribution was evaluated using Shapiro-Wilk tests. The homogeneity of variances was assessed using Levene tests. Pairs of independent groups were compared using independent samples t-tests with Bootstrap results, and Mann-Whitney U-tests with Monte Carlo results. Categorical variables were compared using Pearson $\chi^{2}$ tests with exact results. Correlations between KISS-1 and age, number of births, parity, and abortus were assessed using Spearman rho tests. The sensitivity and specificity of relationships between classifications based on cutoff values calculated according to the number of abortions and KISS-1 variables for the 2 groups of patients, and the actual classification were analyzed using receiver operating characteristics curves. Diagnostic-dependent variables with explanatory variables were estimated using logistic regression analysis, K-Nearest Neighbor, and Decision Tree. However, the results were not significant and are not shown. Quantitative variables are expressed as mean \pm standard deviation, range (maximum-minimum), and medians. Categorical variables are expressed as number (\%). The variables were analyzed at $95 \%$ confidence intervals, and $P<0.05$ was considered statistically significant.

Table 1. Comparison of demographic data and KISS- 1 levels between the 2 groups

\begin{tabular}{|c|c|c|c|c|}
\hline Characteristics & Optional abortion $(n=24)$ & Abortus $(n=27)$ & Total $(n=51)$ & $P$-value \\
\hline Age & $31.54 \pm 4.95(41-24)$ & $31.89 \pm 7.37(43-19)$ & $31.73 \pm 6.29(43-19)$ & 0.839 \\
\hline Parity & $2(8-1)$ & $2(7-1)$ & $2(8-1)$ & 0.893 \\
\hline KISS-1 & $3(3-2)$ & $2(3-2)$ & $3(3-2)$ & 0.004 \\
\hline \multicolumn{5}{|l|}{ Gravida } \\
\hline Primigravid & $8(33.3)$ & $8(29.6)$ & $16(31.4)$ & 1 \\
\hline Multigravid & $16(66.7)$ & $19(70.4)$ & $35(68.6)$ & \\
\hline \multicolumn{5}{|l|}{ Previous abortion } \\
\hline None & $21(87.5)$ & $19(70.4)$ & $40(78.4)$ & 0.182 \\
\hline 1 & $3(12.5)$ & $8(29.6)$ & $11(21.6)$ & \\
\hline
\end{tabular}

Values are presented as mean \pm standard deviation, median (maximum-minimum), or number (\%). Data were compared using independent samples $t$-tests (Bootstrap), Mann-Whitney U-tests (Monte Carlo), and Pearson $\chi^{2}$ tests (exact). 


\section{Obstetrics \& Gynecology Science}

Eser Colak, et al. Placental KISS-1 in first trimester

\section{Results}

Table 1 shows the demographic characteristics of the patients. Mean age (control vs. study group: $31.54 \pm 4.95$ vs. $31.89 \pm 7.37$ years), gravida status, parity number, previous abortions and gestational weeks ( $P=0.072)$ did not signifi-

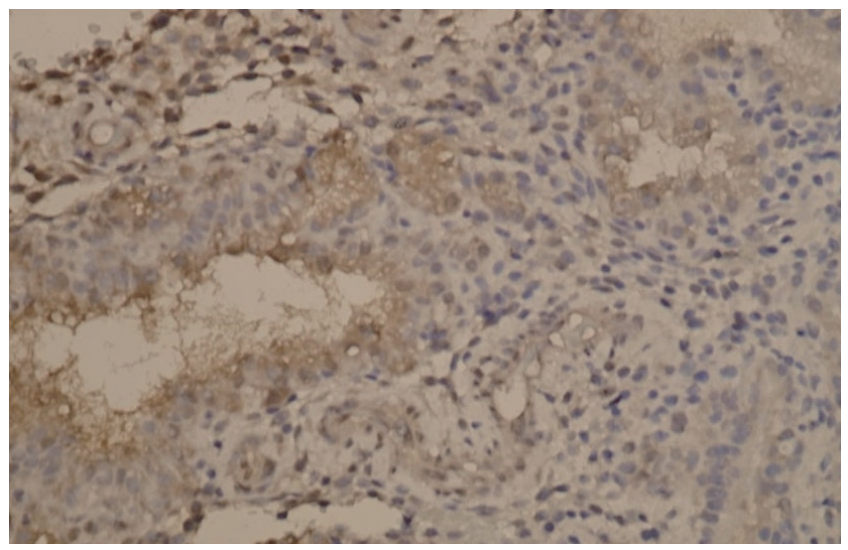

Fig. 1. Weak (+) staining intensity.

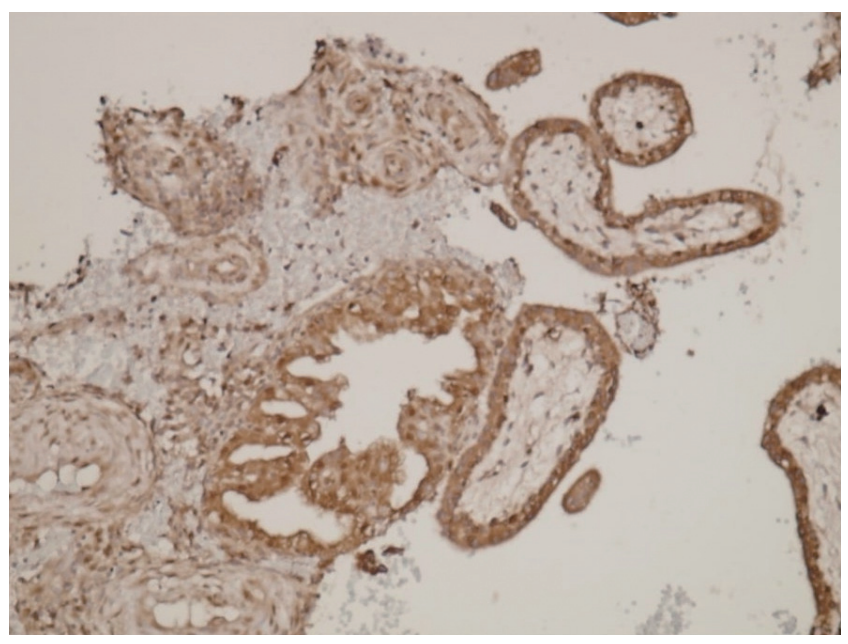

Fig. 2. Strong $(+++)$ intensity of staining. cantly differ between the 2 groups. These finding confirmed the homogeneous distribution of demographic characteristics within each group.

Median values of placental KISS-1 expression significantly differed between the two groups. Median values for staining intensity indicating KISS-1 expression in the control and study groups were 3 (strong) and 2 (moderate), respectively $(P=0.004$; Table 1). Fig. 1 shows a loss of KISS-1 in expression in the glandular epithelium and decidualized stromal cells. Fig. 2 shows strong KISS-1 staining intensity in the glandular epithelium_and decidualized stromal cells.

The effects of previous abortions on placental KISS-1 expression were significantly lower in the group with elective termination $(P=0.002)$, but did not significantly differ in the group with spontaneous abortion (Table 2).

We determined a cutoff value of 2 for KISS-1 expression based on the areas under receiver operator characteristics curves. The sensitivity and specificity were $59.3 \%$ and $83.3 \%$, respectively (Table 3, Fig. 3).

The expression of KISS-1 was significantly lower in the patients with, than without a history of abortion $(P<0.001$; Table 4).

Table 2. Investigation of the relationship between KISS-1 levels and previous abortion in both groups

\begin{tabular}{lccc}
\hline Previous abortion & No. & KISS-1 & $P$-value \\
\hline $\begin{array}{l}\text { Optional abortion } \\
\text { No }\end{array}$ & 21 & $3(3-2)$ & 0.002 \\
Yes & 3 & $2(2-2)$ & \\
Abortus & & & \\
No & 19 & $2(3-2)$ & 1 \\
Yes & 8 & $2(3-2)$ & \\
\hline
\end{tabular}

Values are presented as median (maximum-minimum). Data were compared using Mann-Whitney U-tests (Monte Carlo).

Table 3. Determination of cut-off, sensitivity and specificity values for KISS-1

\begin{tabular}{lcccc}
\hline Cut-off & Optional abortion $(\mathbf{n}=\mathbf{2 4})$ & Abortus $(\mathbf{n}=\mathbf{2 7})$ & AUC $(\mathbf{9 5} \% \mathrm{Cl})$ & $\boldsymbol{P}$-value \\
\hline KISS-1 & $20(83.3)^{\mathrm{a})}$ & $11(40.7)$ & $0.713(0.856-0.570)$ & 0.004 \\
$>2$ & $4(16.7)$ & $16(59.3)^{b)}$ & & \\
$<2$ & &
\end{tabular}

Values are presented as number (\%). Data were analyzed using ROC (Honley \& Mc Nell - Youden index J).

AUC, area under the ROC curve; $\mathrm{Cl}$, confidence interval; $\mathrm{ROC}$, receiver operating curve.

a) Sensitivity; ${ }^{\text {b) }}$ Specificity. 


\title{
Obstetrics \& Gynecology Science
}

\author{
Vol. 63, No. 4, 2020
}

\section{Discussion}

This study investigated whether placental KISS-1 expression differed between patients with pregnancy losses and those who had elective pregnancy terminations within $\leq 10$ weeks of the first trimester.

Serum kisspeptin values during early gestation are found to be associated with abortive imminence, poor obstetric outcomes such as preterm birth, preeclampsia, and intrauterine growth retardation [16]. The same study also associated kisspeptin with recurrent pregnancy losses, indicating that serum kisspeptin could serve as a marker of placental function [12]. However, comparative information about KISS-1 expression between healthy pregnancies and non-recurrent abortion is scarce.

Our results showed significantly lower placental KISS-1 expression in patients with first-trimester pregnancy losses than in those requesting elective terminations. As far as we are aware, this is the first comparison of KISS-1 in materials de-

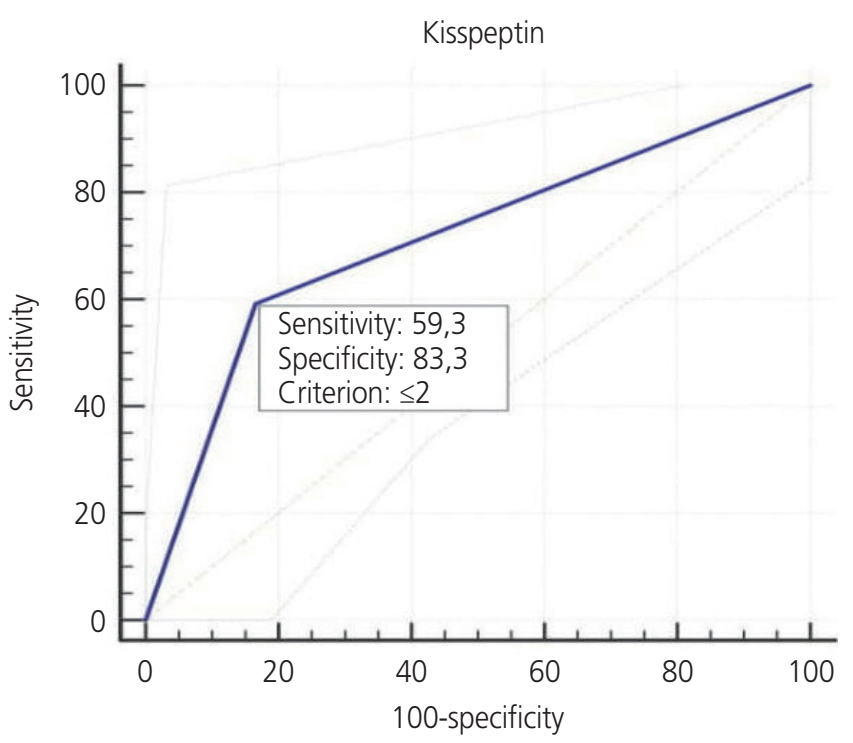

Fig. 3. Sensitivity and specificity for KISS-1. rived from non-recurrent spontaneous abortions and elective pregnancy terminations.

Kisspeptin has many effects on the physiology of puberty, the neuroendocrine system, reproductive

physiology, and it plays a regulatory role in syncytiotrophoblasts $[9,10]$. Kisspeptin and its receptor system are expressed during implantation and placentation in the endometrial and placental tissues of various species, including humans other. A study of hysterectomy materials obtained from non-pregnant female dogs and dogs at different stages of pregnancy found a significant increase in KP-10 levels after implantation and around the middle of pregnancy [17]. Another study found higher levels of KISS-1R expression in the early placenta, where the invasive capacity of trophoblasts needs to be tightly controlled compared with term placenta in humans [18]. These studies suggested a potential role of the kisspeptin/KISS-1R system in the invasive and migratory properties of trophoblasts.

Levels of kisspeptin and its receptor GPR54 were also associated with spontaneous abortions due to their actions on trophoblast invasion. Wu et al. [12] compared the immunohistochemical expression of kisspeptin in decidua and trophoblasts between women with first-trimester pregnancy loss and those who requested legal termination. Levels of kisspeptin were significantly lower in decidual tissue and trophoblasts from women experiencing first-trimester pregnancy loss than in those requesting legal elective termination. We compared KISS-1 expression in decidual cells and trophoblasts between women with first-trimester pregnancy loss and those with elective pregnancy termination. Levels of KISS-1 expression were significantly lower in decidua and trophoblasts from patients with first-trimester pregnancy losses compared with elective termination. We found significantly lower KISS-1 expression in the group with spontaneous abortion, which supports published findings $[12,19]$. However, unlike the patients in those studies, none of our patients had anamnesis of recurrent pregnancy losses.

Table 4. Correlation analysis for KISS-1

\begin{tabular}{|c|c|c|c|c|c|c|}
\hline \multirow{2}{*}{ KISS-1 } & \multicolumn{2}{|c|}{ Optional abortion } & \multicolumn{2}{|c|}{ Abortus } & \multicolumn{2}{|c|}{ Total } \\
\hline & $r$ & $P$-value & $r$ & $P$-value & $r$ & $P$-value \\
\hline Age & -0.243 & 0.252 & 0.170 & 0.397 & 0.005 & 0.97 \\
\hline Previous abortion & -0.845 & $<0.001$ & -0.043 & 0.832 & -0.544 & $<0.001$ \\
\hline
\end{tabular}

Data assessed using Spearman's $\rho$ test. 


\section{Obstetrics \& Gynecology Science}

Eser Colak, et al. Placental KISS-1 in first trimester

In contrast, we identified a negative correlation between KISS-1 expression and abortion in our cohort. The expression of KISS-1 did not significantly differ between patients with spontaneous abortion with and without a history of abortion. However, KISS-1 expression was significantly lower among the women with, than without a history of abortion who requested legal termination. We speculate that previous abortions did not change the KISS-1 expression value in the group with spontaneous abortion because they already had low expression, whereas previous abortions significantly changed the normal level of KISS-1 expression in women with unwanted pregnancies. This result suggests an association between reduced KISS-1 expression and recurrent abortions. Others have also found higher KISS-1 expression in preterm, than in term pregnancy, indicating that KISS-1 expression gradually decreases as the placenta matures $[20,21]$. Together with these findings, the present results suggested that KISS-1 is mainly expressed in the placenta during early pregnancy.

Kisspeptin concentrations have been determined plasma or serum, but few studies have immunohistochemically assessed kisspeptin expression in tissues. Our results showed that KISS-1 is mainly expressed in villous trophoblasts, and that reduced KISS-1 expression is associated with early pregnancy outcomes.

The limitations of our study are that KISS-1 expression was examined only in trophoblastic tissue, and plasma levels were not simultaneously measured. Future studies should investigate correlations between serum kisspeptin levels and trophoblastic KISS-1 expression. Consequently, whether serum kisspeptin could serve as a predictive or diagnostic marker of miscarriage should be investigated. Low expression of KISS1 might be the result of abortions. Therefore, KISS-I expression should be analyzed before and after elective abortion to clarify a cause-and-effect relationship. In addition, detecting KISS-1 mRNA using RT-PCR might be useful to precisely determine whether the KISS-1 gene is affected. Our study did not include patients with recurrent pregnancy loss, which differed from previous similar studies. The reason is that many etiologies can cause pregnancy loss, and a kisspeptin deficiency might function as a cofactor instead of being a direct cause. Thus, we avoided reducing heterogeneity.

In conclusion, we assessed the expression of KISS-1, which is the gene that produces the protein kisspeptin in abortus tissues, and found that KISS-1 plays an important role in the implantation and continuation of pregnancy.

\section{Acknowledgements}

This article was supported by a grant from Baskent University (KA16/132).

\section{Conflict of interest}

No potential conflict of interest relevant to this article was reported.

\section{Ethical approval}

The study protocol was approved by the Institutional Review Board of Baskent University (KA16/132).

\section{Patient consent}

All patients provided written informed consent to participate in the present study.

\section{References}

1. Liu Y, Liu Y, Zhang S, Chen H, Liu M, Zhang J. Etiology of spontaneous abortion before and after the demonstration of embryonic cardiac activity in women with recurrent spontaneous abortion. Int J Gynaecol Obstet 2015;129:128-32.

2. Shih IM, Kurman RJ. New concepts in trophoblastic growth and differentiation with practical application for the diagnosis of gestational trophoblastic disease. Verh Dtsch Ges Pathol 1997;81:266-72.

3. Norwitz ER, Schust DJ, Fisher SJ. Implantation and the survival of early pregnancy. N Engl J Med 2001;345:1400-8.

4. Cignini P, Maggio Savasta L, Gulino FA, Vitale SG, Mangiafico $L$, Mesoraca $A$, et al. Predictive value of pregnancy-associated plasma protein-A (PAPP-A) and free betahCG on fetal growth restriction: results of a prospective 


\section{Obstetrics \& Gynecology Science}

Vol. 63, No. 4, 2020

study. Arch Gynecol Obstet 2016;293:1227-33.

5. Sonek J, Krantz D, Carmichael J, Downing C, Jessup K, Haidar Z, et al. First-trimester screening for early and late preeclampsia using maternal characteristics, biomarkers, and estimated placental volume. Am J Obstet Gynecol 2018;218:126.e1-126.e13.

6. Murray MJ, Lessey BA. Embryo implantation and tumor metastasis: common pathways of invasion and angiogenesis. Semin Reprod Endocrinol 1999;17:275-90.

7. Soundararajan R, Rao AJ. Trophoblast 'pseudo-tumorigenesis': significance and contributory factors. Reprod Biol Endocrinol 2004;2:15.

8. Bilban M, Ghaffari-Tabrizi N, Hintermann E, Bauer S, Molzer S, Zoratti C, et al. Kisspeptin-10, a KiSS-1/ metastin-derived decapeptide, is a physiological invasion inhibitor of primary human trophoblasts. J Cell Sci 2004;117:1319-28.

9. Plant TM. The role of KiSS-1 in the regulation of puberty in higher primates. Eur J Endocrinol 2006;155 Suppl 1:511-6.

10. Roa J, Tena-Sempere M. KiSS-1 system and reproduction: comparative aspects and roles in the control of female gonadotropic axis in mammals. Gen Comp Endocrinol 2007;153:132-40.

11. Hiden U, Bilban M, Knöfler M, Desoye G. Kisspeptins and the placenta: regulation of trophoblast invasion. Rev Endocr Metab Disord 2007;8:31-9.

12. Wu S, Zhang H, Tian J, Liu L, Dong Y, Mao T. Expression of kisspeptin/GPR54 and PIBF/PR in the first trimester trophoblast and decidua of women with recurrent spontaneous abortion. Pathol Res Pract 2014;210:47-54.

13. Sullivan-Pyke C, Haisenleder DJ, Senapati S, Nicolais O, Eisenberg E, Sammel MD, et al. Kisspeptin as a new serum biomarker to discriminate miscarriage from viable intrauterine pregnancy. Fertil Steril 2018;109:137-141. e2.
14. Royal College of Obstetricians and Gynaecologists. The investigation and treatment of couples with reccurent first-trimester and second-trimester miscarriage: greentop guideline No. 17. London: Royal College of Obstetricians and Gynaecologists; 2011.

15. Li L, Tian J, Zhou L, Wu S, Zhang S, Qi L, et al. Role of kisspeptin/GPR54 in the first trimester trophoblast of women with a history of recurrent spontaneous abortion. Int J Clin Exp Pathol 2017;10:8161-73.

16. Kavvasoglu S, Ozkan ZS, Kumbak B, Sımsek M, Ilhan N. Association of kisspeptin-10 levels with abortus imminens: a preliminary study. Arch Gynecol Obstet 2012;285:649-53.

17. Schäfer-Somi S, Ay SS, Kaya D, Sözmen M, Beceriklisoy $H B$, Ağaoğlu AR, et al. Kisspeptin-10 and the G proteincoupled receptor 54 are differentially expressed in the canine pregnant uterus and trophoblast cells. Reprod Domest Anim 2017;52 Suppl 2:123-9.

18. Janneau JL, Maldonado-Estrada J, Tachdjian G, Miran I, Motté N, Saulnier $\mathrm{P}$, et al. Transcriptional expression of genes involved in cell invasion and migration by normal and tumoral trophoblast cells. J Clin Endocrinol Metab 2002;87:5336-9.

19. Park DW, Lee SK, Hong SR, Han AR, Kwak-Kim J, Yang KM. Expression of Kisspeptin and its receptor GPR54 in the first trimester trophoblast of women with recurrent pregnancy loss. Am J Reprod Immunol 2012;67:132-9.

20. Torricelli M, Galleri L, Voltolini C, Biliotti G, Florio P, De Bonis $\mathrm{M}$, et al. Changes of placental Kiss-1 mRNA expression and maternal/cord kisspeptin levels at preterm delivery. Reprod Sci 2008;15:779-84.

21. Terao Y, Kumano S, Takatsu Y, Hattori M, Nishimura A, Ohtaki T, et al. Expression of KiSS-1, a metastasis suppressor gene, in trophoblast giant cells of the rat placenta. Biochim Biophys Acta 2004;1678:102-10. 